\title{
COMBINING ABILITY AND HETEROSIS STUDIES USING DIFFERENT CMS SOURCES IN HYBRID RICE BREEDING
}

\author{
REDA A. M. \\ Rice Research \& Training Center (RRTC), Field Crops Research Institute, ARC, Egypt.
}

(Manuscript received 6 April 2015)

\begin{abstract}
$\mathrm{T}$ his investigation was carried out during 2013 and 2014 rice growing seasons at the Experimental Farm of Agriculture Research Station, Sakha, Kafr El-Sheikh, Egypt, to study the magnitude of genotypic variation, the magnitude of both general and specific combining abilities (GCA \& SCA) and their interaction with the two years as two different environmental conditions, estimation of the genetic parameters and broad and narrow senses heritability and the potentiality of heterosis expression for agronomic and yield and its components characters. Hybrids with a yield advantage of $>1.5 \mathrm{t} /$ ha over the highest yielding check variety Giza 178R were considered as promising. Among 12 hybrid combinations tested, six were most promising with mean performance of grain yield ranging from $11.62 \mathrm{t} / \mathrm{ha}$ for large stigma A/Giza 182R to $12.99 \mathrm{t} /$ ha for G46A/Giza 178R. The yield over the best local inbred check, Giza 178 ranged from 1.10 to 2.47 ton ha' 1. Among the four cytoplasmic male sterile (CMS) lines, G46A and IR $58025 \mathrm{~A}$ were the best general combiners for grain yield. The restorer, Giza 178R was the best general combiner among testers for grain yield and most studied characters.
\end{abstract}

\section{INTRODUCTION}

Hybrid rice has become a field reality in Egypt. The long cherished goal of developing and using rice hybrids in Egypt agriculture, has been accomplished with the release of Egyptian hybrids for commercial cultivation in 2005. For development of commercially useable hybrids, choice of appropriate parental lines possessing good combining ability, high yield potential, good grain quality, resistance to major pests and diseases is a pre-requisite. Development of new improved parental lines possessing desirable characteristics and evaluation of them are the major activities of heterosis breeding programme (El-Mowafi et al. 2005 and El-Mowafi 2006).

Hybrid rice technology is such one innovative breakthrough that can further increase rice production leading to food security and reduction of poverty in Egypt. Hybrid rice varieties can out yield conventional cultivars by at least $15 \%$ under the same input levels. Hence, this technology can be used to break the current yield plateau in rice, where yield levels of the conventional released cultivars has stabilized (Bastawisi et al. 2003 and El-Mowafi et al. 2005). 
Combining ability provides an important tool for selection of desirable parents and getting required information on gene action controlling desirable traits ( Rastogi et al. 2011). Kempthone (1957) suggested line $x$ tester analysis as faster and more reliable method to estimate combining ability and screen suitable lines for hybridization. The parents with good GCA can be used to obtain hybrid with strong heterosis in all the crosses developed from them and function of the two parents of a hybrid (Yan et al. 2000).

The aim of the present study is to estimate combining ability and standard heterosis ( economic heterosis) for the yield and its components in $F_{1}$ hybrids developed using male sterility-fertility restoration system using cytoplasmic male sterile lines (CMS) and three Egyptian restorer lines.

\section{MATERIALS AND METHODS}

The present investigation was carried out during 2013 and 2014 rice growing seasons at the farm of Agriculture Research Station, Sakha, Kafr El-Sheikh, Egypt.

The experiment comprised hybrid progenies derived from 12 hybrid rice combinations generated through line $x$ tester mating design. Four cytoplasmic male sterile lines (CMS) Viz., IR 58025A (WA) type, IR 68885 A (mutant type), G46A and large stigma, were used as female lines. Three Egyptian restorer varieties Viz., Giza 178R, Giza 181R and Giza 182R were used as pollen parents (testers) (Table 1). Each of the three restorers, Giza 178R, Giza 181R and Giza 182R and the four CMS lines were grown in isolated plots surrounded by sorghum or coen plots with a row ratio of 10A:3R to get hybrid seeds for all the hybrid rice combinations tested.

Table 1. Name, source and origin of cytoplasmic male sterile (CMS) and restorer (R) lines used in the study.

\begin{tabular}{|l|l|c|}
\hline Genotype & \multicolumn{1}{|c|}{ Cytoplasmic source } & Origin \\
\hline CMS lines (female): & & \\
\hline IR 58025 A & CMS line wide abortive (WA) & IRRI \\
\hline IR 58025 B & Maintainer line & \\
\hline IR 68885 A & Mutant IR 62829A & IRRI \\
\hline IR 68885 B & Maintainer line & China \\
\hline G 46 A & CMS lines Gambica & \\
\hline G 46 B & Maintainer line & China \\
\hline Large stigma A & CMS line (Kalinga) & \\
\hline Large stigma B & Maintainer line & \\
\hline Restorer lines (male): & & Egypt \\
\hline Giza 178 R & Restorer & Egypt \\
\hline Giza 181 R & Restorer & Egypt \\
\hline Giza 182 R & Restorer &
\end{tabular}


The $F_{1}$ hybrid combinations along with their respective parents were grown in a randomized block design with four replication. Thirty day-old seedling were transplanted with one seedling hill ${ }^{-1}$ adopting a spacing of $20 \mathrm{~cm}$ between rows and $20 \mathrm{~cm}$ between plants. Each test entry consisted of 10 rows of five meter length.

Observations were recorded on ten plants plot $^{-1}$ taken at random from each entry in each replication for heading date ( days to $50 \%$ flowering), plant height, panicles plant ${ }^{-1}$, panicle length $(\mathrm{cm})$, panicle weight $(\mathrm{g})$, spikelets panicle ${ }^{-1}$ ( bagged and unbagged), filled grains panicle ${ }^{-1}$ ( bagged and unbagged), spikelet fertility \% (bagged and unbagged) and 1000 grain weight $(\mathrm{g})$. Five guarded rows $\left(5 \mathrm{~m}^{2}\right)$ were harvested from each entry in each replication to determine grain yield (ton ha ${ }^{-1}$ ).

The data were subjected to analysis of variances for RCBD as suggested by Panse and Sukhatme (1954) and the analysis of variance for line $x$ tester was carried as suggested by Kempthorne (1957) for random lines representing certain population.

The combined data was also calculated over the two years to test the interaction of the different genetic components with the two years. The test described by Bartlett (1937).

Standard heterosis ( over the best check variety, Giza 178) was calculated for the studied characters.

\section{RESULTS AND DISCUSSION}

Analysis of variance

Analysis of variance (Table 2) revealed highly significant differences among the 19 genotypes (12 hybrids, four CMS lines and three restorers) tested for all studied characters. The parental lines and the hybrids showed highly significant differences for all characters. Parents $V s$ crosses mean square indicated that average of heterosis was significant in all crosses for all studied characters at the two years and across them.

The four interaction types, i.e. genotypes with years, parents with years, hybrids with years and parents vs. hybrids with years were found to be significant for grain yield ton $h^{-1}$. However the three types of interaction, i.e. genotypes, hybrids and parents vs. crosses were found to be significant or highly significant for plant height, panicles plant ${ }^{-1}$, panicle weight, filled grains panicle ${ }^{-1}$ (unbagged) and spikelet fertility \% (unbagged). On the other hand, the interactions of genotypes, parents, hybrids and parents vs. hybrids with years were insignificant for heading date except hybrids, panicle length, spikelets panicle ${ }^{-1}$ (bagged and unbagged), filled grains panicle ${ }^{1}$ (bagged), spikelet fertility \% (bagged) and 1000-grain weight except P vs. hybrids. 
The interaction of parents with years was found to be insignificant for all agronomic and yield and its components characters except yield ton $\mathrm{ha}^{-1}$.

The analysis of variance for general combining ability (GCA) given in table (2) revealed significant differences among CMS lines at both years and across for plant height, panicles plant $^{-1}$, spikelets panicle ${ }^{-1}$ (bagged and unbagged), filled grains panicle $^{-1}$ (bagged and unbagged) at the two years and 1000- grain weight. The testers exhibited significant differences for days to heading at the first year and combined analysis, panicles plant ${ }^{-1}$, spikelets panicle ${ }^{-1}$ (bagged and unbagged) and at the two years for 1000-grain weight.

The line $x$ tester (SCA) variance exhibited significant differences at the two years and their combined analysis for heading date, plant height, panicle length, panicle weight, spikelets panicle ${ }^{-1}$ (bagged and unbagged), filled grains panicle ${ }^{-1}$ (unbagged) spikelet fertility \% (bagged and unbagged) 1000-grain weight and grain yield ton $\mathrm{ha}^{-1}$ characters. On the other hand, the $L \mathrm{x} T$ (SCA) variance showed highly significant at the second year and combined analysis for panicles plant ${ }^{-1}$ and at the first year only for filled grains panicle (bagged) characters indicating the importance of dominance or non additive variance in their expression (Table 2).

General Combining Ability effects (GCA)

The estimates values of GCA effects gi for the studied characters are presented in table (3). Among CMS line IR68885A was best combiner for earliers (due to having negative and highly significant gca for days to heading) and short stature of plant. The CMS line IR 58025A exhibited highly significant positive gi $\hat{i}^{\wedge}$ effects and best combiner for panicle length $(\mathrm{cm})$, panicle weight $(\mathrm{g})$, spikelets panicle ${ }^{-1}$ (bagged and unbagged), filled grains panicle ${ }^{-1}$ ( bagged and unbagged) and good combiner for yield $t / h a$. G46A was good combiner for panicle weight $(\mathrm{g})$, filled grains panicle ${ }^{-1}$, 1000-grain weight and best combiner for spikelet fertility \% ( bagged and unbagged) and grain yield ton $\mathrm{ha}^{-1}$. Large stigma $\mathrm{A}$ was good combiner for days to heading, spikelets panicle ${ }^{-1}$, filled grains panicle ${ }^{-1}$ and best combiner for 1000 -grain weight.

Among testers (Table 3), Giza 178R was the best general combiner for plant height, panicle weight (g), spikelets panicle ${ }^{-1}$, filled grains panicle ${ }^{-1}$, spikelet fertility $\%$ and yield ton ha ${ }^{-1}$ and good combiner for days to heading. On the other hand, the restorer variety Giza 181R was good combiner for panicle length and good combiner for panicles plant ${ }^{-1}$ and 1000 -grain weight. Giza $182 \mathrm{R}$ was best combiner for days to heading, panicles plant ${ }^{-1}$ and 1000 -grain weight and good combiner for panicle weight character.

Specific Combining Ability effects (Sij) 
The estimates of $\mathrm{Sij}$ effects revealed that none of the hybrid were consistently superior for all the characters (Table 4). The hybrid combination IR 68885A / Giza 178R was superior or ranking the first in earliness and significant for grain yield ton $\mathrm{ha}^{-1}$. With respect to heading date and plant height five and three hybrid combinations showed negative and significant effects in the desired direction for the two traits, respectively. For panicle length, three hybrids had superior $\mathrm{Sij}$ effects, five hybrids for spikelets panicle ${ }^{-1}$ ( bagged and unbagged), five hybrid combination for filled grains panicle ${ }^{-1}$, three for spikelet fertility $\%$, three for 1000 grain weight and five for grain yield ton ha ${ }^{-1}$. The hybrid combinations IR68885A / Giza 181R, G46A/Giza 178R, IR68885A/Giza 181R, and large Stigma A/ Giza 182R were best combination for most of studied characters.

Genetic parameters:

Line $\mathrm{x}$ tester mating design were developed partition genetic variance into its components. The total genetic variance was divided to lines, testers and line $x$ tester interaction components. The first portion of variance i.e., line and tester estimate additive genetic variance while the second portion line by tester interaction estimate non-additive genetic variance including dominance. The estimates of genetic parameters viz., additive variance $\left(\sigma^{2} A\right)$, non-additive including dominance variance $\left(\sigma^{2} D\right)$, environmental variance $\left(\sigma^{2} E\right)$, genotypic variance $\left(\sigma^{2} G\right)$, phenotypic variance $\left(\sigma^{2} p\right)$, brood sense heritability $\left(h^{2} b \%\right)$ and narrow sense heritability $\left(h^{2} n \%\right)$ were obtained and the results are shown in Table (5). In the same time, the relative importance of GCA \% and relative importance of SCA \% for all the studied characters are presented in the same table.

The results reveled that the estimates of the additive variance $\left(\sigma^{2} A\right)$ and the relative importance of GCA \% for days to heading, plant height, tillers plant ${ }^{-1}$, spikelets panicle ${ }^{-1}$ (bagged and unbagged), filled grains panicle ${ }^{-1}$ (bagged and unbagged) and 1000-grain weight was higher than those dominance variance $\left(\sigma^{2} D\right)$. These findings indicated the former characters were largely governed by additive gene action. On the other hand, the results cleared that the estimates of the non-additive $\left(\sigma^{2} D\right)$ and the relative importance of SCA\% for spikelet fertility \% (bagged and unbagged), panicle length, panicle weight and grain yield was higher than those additive variance $\left(\sigma^{2} \mathrm{~A}\right)$ and relative importance of GCA\%, indicated that the former characters were largely governed by non-additive gene action. Many authors obtained similar results which indicated to importance of the additive gene action among them El-Mowafi (2001a), El Mowafi and Abou Shousha (2003), El Mowafi et al. (2005) and Abd Allah (2008). On the other hand, the importance of the non-additive or dominance gene action for the inheritance of the former characters were reported by Babu and Reddy (2002), El Refaee (2002), El Mowafi et al. (2005) and El Diasty et al. (2008). 
Concerning heritability estimates in brood sense $\left(h^{2} b\right)$, the results indicated that the values estimated of $h^{2} b$ were high for most of the studied characters. However, heritability estimates in the narrow sense $\left(h^{2} n\right)$ were high for days to heading $(50.53 \%)$, plant height $(78.42 \%)$, tillers plant ${ }^{-1}(75.01 \%)$, spikelets panicle ${ }^{-1}$ under bagged $(69.17 \%)$, spikelets panicle ${ }^{-1}$ under unbagged $(69.49 \%)$, filled grains panicle $^{-1}$ under bagged and unbagged $(61.18 \%$ and $65.31 \%)$ and 1000 grain weight $(87.93 \%)$. These results indicated that a major part of the total genotypic variances was additive in nature and are in general agreement with those reported by El Mowafi and Abou Shoush (2003), El-Mowafi et al. (2005) and Abd Allah (2008). On the hand, heritability values in narrow sense were relatively low for spikelet fertility $\%$ with bagged and unbagged (8.86 and $29.95 \%)$, panicle length $(-15.02 \%)$, panicle weight $(38.80 \%)$, and grain yield character $(0.29 \%)$. The results also illustrated that a major part of the total phenotypic variance was due to non-additive or dominance genetic for the former characters. Accordingly, it was expected that an effective heterosis on breeding program or hybrid rice breeding for these characters. Similar results were obtained by El Refaee (2002), Abd Allah (2008) and El-Badawi (2009).

\section{MEAN PERFORMANCE}

Mean performance of seven parental lines (four CMS and three restorers) and their 12 hybrid combinations of line $\mathrm{x}$ tester for the ten studied characters are presented in table (6). For days to heading, theF $F_{1}$ mean values of 11 hybrid combinations were towards the lower parents ( early flowering parents), while one only tended to higher parents (late flowering parents). Days to heading ranged from 94.4 days to 112.8 days. The hybrids IR68885A / Giza 178R, large stigma A / Giza $182 \mathrm{R}$ and IR68885A / Giza 182R were noted to be early maturing hybrid combinations, while IR58025A / Giza 181R was a late maturing hybrid. Early maturing hybrids are desirable as they produce more yield per day. Highest plant height were observed in G46A / Giza 182R $(116.0 \mathrm{~cm})$, G46A / Giza 178R $(113.0 \mathrm{~cm})$ and IR 58025A / Giza 181R (111.4cm) and lowest in IR68885A / Giza 178R $(97.7 \mathrm{~cm})$ and IR68885A / Giza 182R (98.6cm). The hybrid IR68885A / Giza 182R was exhibited highest panicles plant ${ }^{-1}$ and least was found in large stigma A / Giza 178R ranged from 24.8 to 17.6 panicles. While the check variety Giza 178 exhibited 20.8 panicles. Hybrids are generally characterized by having larger panicles indicating their efficiency in partitioning of assimilates to reproductive parts ( Neelam et al. 2009). Panicle length character was varied from $25.3 \mathrm{~cm}$ ( in larg stigmaA / Giza 181R) to $28.7 \mathrm{~cm}$ (in IR58025A / Giza 181R). 
Table 2. Estimates of the mean squares of ordinaries analysis for agronomic and yield component characters in the two years and their combined data.

\begin{tabular}{|c|c|c|c|c|c|c|c|c|c|c|c|c|c|c|c|c|c|}
\hline \multirow{2}{*}{ S.O.V. } & \multicolumn{2}{|c|}{ d. f. } & \multicolumn{3}{|c|}{ Heading date } & \multicolumn{3}{|c|}{ Plant height } & \multicolumn{3}{|c|}{ Panicles plant ${ }^{-1}$} & \multicolumn{3}{|c|}{ Panicle length } & \multicolumn{3}{|c|}{ Panicle weight } \\
\hline & Single & Comb. & $Y_{1}$ & $Y_{2}$ & Comb. & $Y_{1}$ & $Y_{2}$ & Comb. & $Y_{1}$ & $Y_{2}$ & Comb. & $Y_{1}$ & $Y_{2}$ & Comb. & $Y_{1}$ & $Y_{2}$ & Comb. \\
\hline Years (Y) & 1 & 1 & - & - & 1.7 & - & - & 0.71 & - & - & $63.31^{* *}$ & - & - & $0.024^{\text {n.s }}$ & - & - & $0.07^{* *}$ \\
\hline Reps/ (Y) & 3 & 6 & - & - & 0.7 & - & - & 0.75 & - & - & 2.13 & - & - & 0.149 & - & - & 0.02 \\
\hline Genotypes(G) & 18 & 18 & $289.3^{* *}$ & $296.7^{* *}$ & $584.6^{* *}$ & $177.0^{* *}$ & $153.9^{* *}$ & $319.9^{* *}$ & $23.01^{* *}$ & $52.42^{* *}$ & $64.20^{* *}$ & $23.01^{* *}$ & $52.42^{* *}$ & $30.82^{* *}$ & $2.71^{* *}$ & $2.28^{* *}$ & $4.88^{* *}$ \\
\hline Parent (P) & 6 & 6 & $6407^{* *}$ & $685.3^{* *}$ & $1324.7^{* *}$ & $165.7^{* *}$ & $182.8^{* *}$ & $346.3^{* *}$ & $31.15^{* *}$ & $32.00^{* *}$ & $60.70^{* *}$ & $31.15^{* *}$ & $32.00^{* *}$ & $18.81^{* *}$ & $0.95^{* *}$ & $0.95^{* *}$ & $1.91^{* *}$ \\
\hline Grosses (C) & 11 & 11 & $81.2^{* *}$ & $70.1^{* *}$ & $149.7^{* *}$ & $164.9^{* *}$ & $128.4^{* *}$ & $276.9^{* *}$ & $18.38^{* *}$ & $49.56^{* *}$ & $54.88^{* *}$ & $18.38^{* *}$ & $49.56^{* *}$ & $7.78^{* *}$ & $2.52^{* *}$ & $1.69^{* *}$ & $4.03^{* *}$ \\
\hline P.Vs. (C) & & 1 & $469.9^{* *}$ & $458.6^{* *}$ & $928.4^{* *}$ & $378.8^{* *}$ & $261.5^{* *}$ & $634.8^{* *}$ & $25.06^{* *}$ & $206.4^{* *}$ & $187.68^{* *}$ & $25.06^{* *}$ & $206.4^{* *}$ & $356.36^{* *}$ & $15.35^{* *}$ & $16.75^{* *}$ & $32.08^{* *}$ \\
\hline$G \times Y$ & & 18 & - & - & 1.5 & - & - & $10.9^{* *}$ & - & - & $11.23^{* *}$ & - & - & $0.29^{n . s}$ & - & - & $0.11^{*}$ \\
\hline$P \times Y$ & & 6 & - & - & 1.3 & - & - & 2.2 & - & - & 2.45 & - & - & $0.56^{\mathrm{n} . \mathrm{s}}$ & - & - & 0.00 \\
\hline$C \times Y$ & & 11 & - & - & $1.6^{*}$ & - & - & $16.3^{* *}$ & - & - & $13.06^{* *}$ & - & - & $0.16^{\mathrm{n} . \mathrm{s}}$ & - & - & $0.18^{* *}$ \\
\hline P.Vs. $C \times Y$ & & 1 & - & - & 0.04 & - & - & $5.4^{*}$ & - & - & $43.82^{* *}$ & - & - & $0.00^{n . s}$ & - & - & $0.02^{*}$ \\
\hline Lines (GCA) & 3 & 3 & 70.7 & 73.2 & 143.8 & $502.2^{* *}$ & $396.8^{* *}$ & $876.3^{* *}$ & $62.33^{* *}$ & $122.9^{*}$ & $164.72^{* *}$ & $62.33^{* *}$ & $122.9^{*}$ & $5.71^{\mathrm{n} . \mathrm{s}}$ & 4.58 & 3.24 & 7.58 \\
\hline $\begin{array}{l}\text { Testers } \\
\text { (GCA) }\end{array}$ & 2 & 2 & $227.5^{*}$ & 172.6 & $398.2^{*}$ & 18.5 & 41.6 & 54.3 & $6.27^{* *}$ & 17.33 & 21.22 & $6.27^{* *}$ & 17.33 & $7.72^{\mathrm{n} . \mathrm{s}}$ & 2.58 & 0.81 & 3.13 \\
\hline $\mathrm{L} \times \mathrm{T}(\mathrm{SCA})$ & 6 & 6 & $37.7^{* *}$ & $34.3^{* *}$ & $69.8^{* *}$ & $44.9^{* *}$ & $23.1^{* *}$ & $51.5^{* *}$ & 0.44 & $23.64^{* *}$ & $11.19^{* *}$ & 0.44 & $23.64^{* *}$ & $8.83^{* *}$ & $1.47^{* *}$ & $1.20^{* *}$ & $2.55^{* *}$ \\
\hline$L x Y$ & & 3 & - & - & 1.6 & - & - & 22.7 & - & - & $20.50^{*}$ & - & $\begin{array}{c}- \\
20.50^{*}\end{array}$ & $0.09^{n . s}$ & - & - & 0.24 \\
\hline$T \times Y$ & & 2 & - & - & 0.2 & - & - & 5.8 & - & - & 2.38 & - & -2.38 & $0.33^{n . s}$ & - & - & 0.26 \\
\hline$L \times T \times Y$ & & 6 & - & - & 1.9 & - & - & $16.6^{*}$ & - & - & $12.89^{* *}$ & - & $-12.89^{* *}$ & $0.16^{n . s}$ & - & - & 0.12 \\
\hline Error & 54 & 108 & 0.90 & 0.74 & 0.8 & 3.20 & 3.59 & 3.55 & 1.09 & 1.11 & 1.15 & 1.09 & 1.15 & 0.185 & 0.09 & 0.03 & 0.05 \\
\hline C.V \% & & & 0.95 & 0.86 & 0.91 & 1.69 & 1.80 & 1.78 & 5.39 & 4.22 & 5.38 & 5.39 & 5.38 & 1.70 & 7.22 & 4.11 & 4.90 \\
\hline
\end{tabular}

$*$ and ${ }^{*}$ significant at $5 \%$ and $1 \%$ probability levels, respectively. 
Cont. Table 2. Estimates of the mean squares of ordinaries analysis for agronomic and yield component characters in the two years and their combined data.

\begin{tabular}{|c|c|c|c|c|c|c|c|c|c|c|c|c|}
\hline \multirow{3}{*}{ Genotypes } & \multicolumn{6}{|c|}{ Spikelets panicle $^{-1}$} & \multicolumn{6}{|c|}{ Filled grains panicle ${ }^{-1}$} \\
\hline & \multicolumn{3}{|c|}{ Bagged } & \multicolumn{3}{|c|}{ Unbagged } & \multicolumn{3}{|c|}{ Bagged } & \multicolumn{3}{|c|}{ Unbagged } \\
\hline & $Y_{1}$ & $\mathrm{Y}_{2}$ & Comb. & $Y_{1}$ & $Y_{2}$ & Comb. & $Y_{1}$ & $Y_{2}$ & Comb. & $Y_{1}$ & $Y_{2}$ & Comb. \\
\hline Years $(Y)$ & - & - & 31.3 & - & - & $222.7^{*}$ & - & - & 3844.3 & - & - & 55.0 \\
\hline Reps/ (Y) & - & - & 23.4 & - & - & 85.1 & - & - & 5230.5 & - & - & 66.1 \\
\hline Genotypes(G) & $4863.0^{* *}$ & $4159.9^{* *}$ & $8967.1^{* *}$ & $4181.2^{* *}$ & $4306.8^{* *}$ & $8455.3^{* *}$ & $3182.8^{* *}$ & $2795.6^{* *}$ & $5908.6 * *$ & $3284.2^{* *}$ & $3064.8^{* *}$ & $6250.6^{* *}$ \\
\hline Parent $(P)$ & $5661.4^{* *}$ & $4766.9^{* *}$ & $10363.2^{* *}$ & $4661.5^{* *}$ & $4736.0^{* *}$ & $9384.5^{* *}$ & $2685.1^{* *}$ & $2478.5^{* *}$ & $5104.9^{* *}$ & $2681.2^{* *}$ & $2664.7^{* *}$ & $5327.2^{* *}$ \\
\hline Grosses (C) & $4234.6^{* *}$ & $3404.7^{* *}$ & $7588.3^{* *}$ & $3734.5^{* *}$ & $3859.8^{* *}$ & $7548.3^{* *}$ & $3448.8^{* *}$ & $2836.7^{* *}$ & $6206.4^{* *}$ & $3647.2^{* *}$ & $3429.7^{* *}$ & $6937.4^{* *}$ \\
\hline P.Vs. (C) & $6985.5^{* *}$ & $8825.2^{* *}$ & 15757.0 & $6213.2^{* *}$ & $6648.7^{* *}$ & $12858.2^{* *}$ & $3242.9^{* *}$ & $4245.4^{* *}$ & $7454.5^{* *}$ & $2909.8^{* *}$ & $1451.4^{* *}$ & $4235.7^{* *}$ \\
\hline GxY & - & - & 55.9 & - & - & 32.6 & - & - & 69.8 & - & - & $98.4^{*}$ \\
\hline$P X Y$ & - & - & 65.2 & - & - & 13.1 & - & - & 58.7 & - & - & 18.7 \\
\hline$C X Y$ & - & - & 51.1 & - & - & 45.9 & - & - & 79.1 & - & - & $139.5^{*}$ \\
\hline P.Vs. CxY & - & - & 53.7 & - & - & 3.7 & - & - & 33.8 & - & - & $125.5^{*}$ \\
\hline Lines (GCA) & $11774.8^{*}$ & $1006.9^{*}$ & $21825.8^{* *}$ & $10630.9^{*}$ & $11071.7^{*}$ & $21613.4^{* *}$ & $8742.0^{* *}$ & $7865.9^{*}$ & 16474.1 & $9576.5^{*}$ & $8704.7^{*}$ & 17887.2 \\
\hline Testers (GCA) & 1156.7 & 536.7 & $1605.2^{* *}$ & 871.3 & 1095.3 & $1940.1^{* *}$ & 1434.3 & 594.8 & 1896.2 & 1745.6 & 1935.4 & 3674.4 \\
\hline $\mathrm{LxT}(\mathrm{SCA})$ & $1490.5^{* *}$ & $1004.6^{*}$ & $2463.8^{* *}$ & $1240.6^{* *}$ & $1175.2^{* *}$ & $2385.1^{* *}$ & $1437.8^{* *}$ & 1069.4 & 2509.3 & $1316.4^{* *}$ & $1288.8^{* *}$ & $2550.2^{* *}$ \\
\hline Lxy & - & - & 65.9 & - & - & 89.3 & - & - & 133.8 & - & - & 397.0 \\
\hline$T X Y$ & - & - & 88.2 & - & - & 26.4 & - & - & 132.9 & - & - & 6.6 \\
\hline LXTXY & - & - & 31.3 & - & - & 30.7 & - & - & 33.9 & - & - & 55.0 \\
\hline Error & 45.7 & 42.9 & 45.3 & & & 53.8 & 50.2 & 35.6 & 42.7 & 51.0 & 41.1 & 45.9 \\
\hline C.V \% & 3.87 & 3.77 & 3.86 & 4.13 & 4.22 & 4.19 & 4.98 & 4.25 & 4.95 & 4.72 & 4.27 & 4.50 \\
\hline
\end{tabular}

* and $* *$ significant at $5 \%$ and $1 \%$ probability levels, respectively. 
Cont. Table 2. Estimates of the mean squares of ordinaries analysis for agronomic and yield component characters (combined analysis).

\begin{tabular}{|c|c|c|c|c|c|c|c|c|c|c|c|c|}
\hline \multirow{3}{*}{ Genotypes } & \multicolumn{6}{|c|}{ Spikelet fertility \% } & \multirow{2}{*}{\multicolumn{3}{|c|}{ 1000-grain weight (g) }} & \multirow{2}{*}{\multicolumn{3}{|c|}{ Grain yield ton ha-1 }} \\
\hline & \multicolumn{3}{|c|}{ Bagged } & \multicolumn{3}{|c|}{ Unbagged } & & & & & & \\
\hline & $Y_{1}$ & $Y_{2}$ & Comb. & $Y_{1}$ & $Y_{2}$ & Comb. & $Y_{1}$ & $Y_{2}$ & Comb. & $Y_{1}$ & $Y_{2}$ & Comb. \\
\hline Years $(Y)$ & - & - & $13.9^{* *}$ & - & - & $115.9^{* *}$ & - & - & 0.10 & - & - & 0.01 \\
\hline Reps/ (Y) & - & - & 3.4 & - & - & 2.5 & - & - & 0.09 & - & - & 0.03 \\
\hline Genotypes(G) & $50.5^{* *}$ & $47.1^{* *}$ & $93.3^{* *}$ & $70.8^{* *}$ & $90.0^{* *}$ & $146.4^{* *}$ & $30.38^{* *}$ & $33.45^{* *}$ & $63.68^{* *}$ & $16.56^{* *}$ & $15.40^{* *}$ & $31.55^{* *}$ \\
\hline Parent $(P)$ & $47.1^{* *}$ & $60.6^{* *}$ & $104.6^{* *}$ & $51.7^{* *}$ & $79.4^{* *}$ & $127.6^{* *}$ & $42.32^{* *}$ & $48.70^{* *}$ & $90.82^{* *}$ & $15.79^{* *}$ & $11.20^{* *}$ & $26.71^{* *}$ \\
\hline Grosses (C) & $48.6^{* *}$ & $37.1^{* *}$ & $80.8^{* *}$ & $75.9^{* *}$ & $60.1^{* *}$ & $119.4^{* *}$ & $19.54^{* *}$ & $19.77^{* *}$ & $39.20^{* *}$ & $10.84^{* *}$ & $11.79^{* *}$ & $21.96^{* *}$ \\
\hline P.Vs. (C) & $91.7^{* *}$ & $76.3^{* *}$ & $167.6^{* *}$ & $129.1^{* *}$ & $483.5^{* *}$ & $556.2^{* *}$ & $78.08^{* *}$ & $92.39^{* *}$ & $170.17^{* *}$ & $84.16^{* *}$ & $80.30^{* *}$ & $166.03^{* *}$ \\
\hline $\mathrm{G} \times Y$ & - & - & 4.3 & - & - & $14.5^{* *}$ & - & - & 0.15 & - & - & $0.41^{* *}$ \\
\hline$P X Y$ & - & - & 3.2 & - & - & 3.5 & - & - & 0.20 & - & - & $0.28^{*}$ \\
\hline$C X Y$ & - & - & 4.8 & - & - & $16.6^{* *}$ & - & - & 0.11 & - & - & $0.67^{* *}$ \\
\hline P.Vs. CXY & - & - & 0.4 & - & - & $56.4^{* *}$ & - & - & $0.30^{*}$ & - & - & $1.57^{* *}$ \\
\hline Lines (GCA) & 82.2 & 40.8 & 113.1 & 128.1 & 49.7 & 142.2 & $55.69^{* *}$ & $55.17^{* *}$ & $110.79^{*}$ & 16.52 & 17.61 & 31.89 \\
\hline Testers (GCA) & 49.6 & 15.8 & 59.0 & 103.1 & 96.3 & 189.7 & $15.90^{*}$ & $18.81^{*}$ & 34.63 & 4.52 & 4.65 & 8.29 \\
\hline $\mathrm{LxT}(\mathrm{SCA})$ & $31.4^{* *}$ & $42.4^{* *}$ & $72.0^{* *}$ & $40.8^{* *}$ & $53.1^{* *}$ & 84.6 & $2.67^{* *}$ & $2.40^{* *}$ & $4.93^{* *}$ & $10.10^{* *}$ & $11.26^{* *}$ & 21.56 \\
\hline$L x Y$ & - & - & 9.9 & - & - & $35.6^{*}$ & - & - & 0.07 & - & - & 2.24 \\
\hline$T \times Y$ & - & - & 6.3 & - & - & 9.7 & - & - & 0.08 & - & - & 0.88 \\
\hline$L \times T \times Y$ & - & - & 1.8 & - & - & 9.3 & - & - & 0.14 & - & - & $0.20^{*}$ \\
\hline Error & 7.1 & 3.4 & 5.1 & 2.6 & 3.6 & 3.1 & 0.15 & 0.29 & 0.13 & 0.16 & 0.12 & 0.058 \\
\hline C.V \% & 3.26 & 2.26 & 2.77 & 1.86 & 2.23 & 2.05 & 8.31 & 10.29 & 8.92 & 3.89 & 3.31 & 2.33 \\
\hline
\end{tabular}

* and $* *$ significant at $5 \%$ and $1 \%$ probability levels, respectively. 
Table 3. Estimates of GCA effects of the CMS lines and restorer lines (R) for agronomic and yield component characters (combined analysis)

\begin{tabular}{|c|c|c|c|c|c|c|c|c|c|c|c|c|c|}
\hline \multirow{2}{*}{ Line } & \multirow{2}{*}{$\begin{array}{l}\text { Heading } \\
\text { date }\end{array}$} & \multirow{2}{*}{$\begin{array}{c}\text { Plant } \\
\text { height }\end{array}$} & \multirow{2}{*}{$\begin{array}{l}\text { Panicles } \\
\text { plant }^{-1}\end{array}$} & \multirow{2}{*}{$\begin{array}{l}\text { Panicle } \\
\text { length }\end{array}$} & \multirow{2}{*}{$\begin{array}{l}\text { Panicle } \\
\text { weight }\end{array}$} & \multicolumn{2}{|c|}{ Spikelets panicle ${ }^{-1}$} & \multicolumn{2}{|c|}{ Filled grains panicle ${ }^{-1}$} & \multicolumn{2}{|c|}{ Spikelet fertility \% } & \multirow{2}{*}{$\begin{array}{c}1000- \\
\text { grain } \\
\text { weight }\end{array}$} & \multirow{2}{*}{$\begin{array}{l}\text { Yield ton } \\
\mathrm{ha}^{-1}\end{array}$} \\
\hline & & & & & & Bagged & Unbagged & Bagged & Unbagged & Bagged & Unbagged & & \\
\hline & Comb. & Comb. & Comb. & Comb. & Comb. & Comb. & Comb. & Comb. & Comb. & Comb. & Comb. & Comb. & Comb. \\
\hline \multicolumn{14}{|l|}{ CMS lines (A): } \\
\hline IR $58025 \mathrm{~A}$ & $3.53^{* *}$ & 0.78 & $-1.21^{* *}$ & $0.72^{* *}$ & $0.54^{* *}$ & $21.21^{* *}$ & $24.84^{* *}$ & $16.21^{* *}$ & $21.98 * *$ & 0.09 & 0.82 & $-0.89^{* *}$ & $0.83^{* *}$ \\
\hline IR $68885 \mathrm{~A}$ & $-2.05^{* *}$ & $-8.43^{* *}$ & $3.29^{* *}$ & $-0.36^{* *}$ & $-0.75^{* *}$ & $-43.63^{* *}$ & $-42.95^{* *}$ & $-38.92^{* *}$ & $-39.90^{* *}$ & $-2.53^{* *}$ & $-2.14^{* *}$ & $-2.56^{* *}$ & $-0.58^{* *}$ \\
\hline G $46 \mathrm{~A}$ & $-0.43 *$ & $5.91^{* *}$ & $0.71^{*}$ & $-0.20^{*}$ & $0.29^{* *}$ & 3.33 & $2.89 *$ & $7.88^{* *}$ & $8.40^{* *}$ & $2.76^{* *}$ & $3.07^{* *}$ & $1.23^{* *}$ & $1.08^{* *}$ \\
\hline Large Stigma A & -0.05 & $1.74^{* *}$ & $-2.79^{* *}$ & -0.16 & -0.08 & $19.08^{* *}$ & $15.22^{* *}$ & $14.83^{* *}$ & $9.52^{* *}$ & -0.32 & $-1.76^{* *}$ & $2.23^{* *}$ & $-1.33^{* *}$ \\
\hline \multicolumn{14}{|l|}{ Restorer lines ( $R$ ) } \\
\hline G. $178 \mathrm{R}$ & $-1.99^{* *}$ & $-1.50^{* *}$ & $-0.94^{* *}$ & $-0.53^{* *}$ & $0.24^{* *}$ & $8.09^{* *}$ & $8.09^{* *}$ & $8.28^{* *}$ & $9.50^{* *}$ & $1.02^{*}$ & $1.92^{* *}$ & $-1.20^{* *}$ & $0.58^{* *}$ \\
\hline G. $181 \mathrm{R}$ & $4.07^{* *}$ & $0.84^{*}$ & 0.41 & $0.44^{* *}$ & $-0.35^{* *}$ & $-5.06 *$ & $-7.44^{* *}$ & $-6.94^{* *}$ & $-9.94^{* *}$ & $-1.54^{* *}$ & $-2.74^{* *}$ & $0.52^{* *}$ & $-0.35^{* *}$ \\
\hline G. $182 \mathrm{R}$ & $-2.08^{* *}$ & 0.66 & 0.53 & 0.09 & $0.11^{*}$ & $-3.03^{*}$ & -0.66 & -1.34 & 0.44 & 0.52 & $0.82^{*}$ & $0.68^{* *}$ & $-0.23^{* *}$ \\
\hline L.S.D. for CMS lines $5 \%$ & 0.38 & 0.86 & 0.65 & 0.19 & 0.11 & 2.79 & 2.87 & 2.76 & 2.96 & 0.91 & 0.88 & 0.18 & 0.18 \\
\hline $1 \%$ & 0.51 & 1.13 & 0.85 & 0.26 & 0.14 & 3.62 & 3.79 & 3.65 & 3.91 & 1.20 & 1.16 & 0.24 & 0.24 \\
\hline L.S.D. for $\mathrm{R}$ lines $\quad 5 \%$ & 0.33 & 0.74 & 0.56 & 0.17 & 0.09 & 2.38 & 2.48 & 2.39 & 2.56 & 0.78 & 0.76 & 0.16 & 0.16 \\
\hline $1 \%$ & 0.44 & 0.98 & 0.74 & 0.22 & 0.12 & 3.14 & 3.28 & 3.16 & 3.38 & 1.04 & 1.01 & 0.21 & 0.21 \\
\hline
\end{tabular}

* and ** significant at 0.05 and 0.01 probability levels, respectively. 
Table 4. Estimates of specific combining ability effects for the $12 F_{1}$ hybrids at the two years and overall data for agronomic and yield characters (combined analysis).

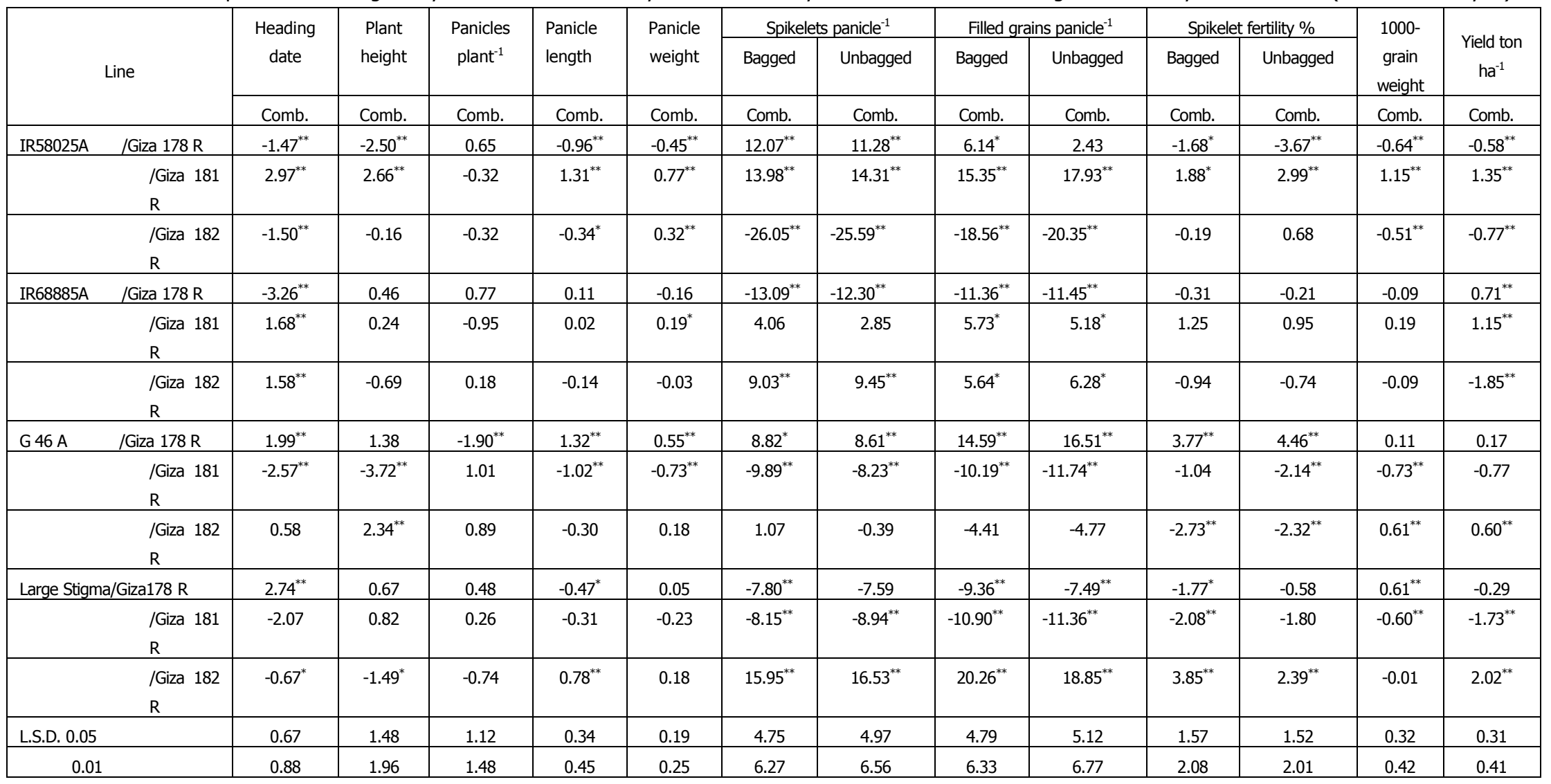

- $\quad$ and $* *$ significant at $5 \%$ and $1 \%$ probability levels, respectively. 
Table 5. Estimates of genetic parameters and heritability in broad senses for agronomic and yield component characters for combined analysis.

\begin{tabular}{|c|c|c|c|c|c|c|c|c|c|c|c|c|c|}
\hline \multirow{3}{*}{ Line } & \multirow{2}{*}{$\begin{array}{l}\text { Heading } \\
\text { date }\end{array}$} & \multirow{2}{*}{$\begin{array}{l}\text { Plant } \\
\text { height }\end{array}$} & \multirow{2}{*}{$\begin{array}{c}\text { Panicles } \\
\text { plant }^{-1}\end{array}$} & \multirow{2}{*}{$\begin{array}{l}\text { Panicle } \\
\text { length }\end{array}$} & \multirow{2}{*}{$\begin{array}{l}\text { Panicle } \\
\text { weight }\end{array}$} & \multicolumn{2}{|c|}{ Spikelets panicle- ${ }^{-1}$} & \multicolumn{2}{|c|}{ Filled grains panicle ${ }^{-1}$} & \multicolumn{2}{|c|}{ Spikelet fertility \% } & \multirow{2}{*}{$\begin{array}{l}1000- \\
\text { grain } \\
\text { weight }\end{array}$} & \multirow{2}{*}{$\begin{array}{l}\text { Yield ton } \\
\text { ha }^{-1}\end{array}$} \\
\hline & & & & & & Bagged & Unbagged & Bagged & Unbagged & Bagged & Unbagged & & \\
\hline & Comb. & Comb. & Comb. & Comb. & Comb. & Comb. & Comb. & Comb. & Comb. & Comb. & Comb. & Comb. & Comb. \\
\hline Additive variances $\left(\sigma^{2} A\right)$ & 13.34 & 34.261 & 6.705 & -0.1645 & 0.227 & 779.9 & 787.25 & 562.70 & 674.15 & 1.306 & 5.685 & 5.34 & 0.1600 \\
\hline $\begin{array}{l}\text { Dominant variance } \\
\left(\sigma^{2} D\right)\end{array}$ & 8.62 & 5.88 & 1.080 & 1.075 & 0.313 & 302.25 & 291.85 & 308.33 & 312.08 & 8.375 & 10.18 & 0.599 & 5.374 \\
\hline $\begin{array}{l}\text { Environmental variance } \\
\left(\sigma^{2} E\right)\end{array}$ & 0.830 & 3.550 & 1.154 & 0.185 & 0.045 & 45.298 & 53.78 & 42.68 & 45.95 & 5.052 & 3.123 & 0.133 & 0.058 \\
\hline $\begin{array}{l}\text { Genotypic variance } \\
\left(\sigma^{2} G\right)\end{array}$ & 21.96 & 40.14 & 7.785 & 0.910 & 0.541 & 1082.15 & 1079.1 & 871.03 & 986.25 & 9.681 & 15.86 & 5.94 & 5.390 \\
\hline $\begin{array}{l}\text { Phenotypic variance } \\
\left(\sigma^{2} P\right)\end{array}$ & 26.45 & 43.69 & 8.939 & 1.095 & 0.585 & 1127.45 & 1132.88 & 975.51 & 1032.18 & 24.733 & 18.98 & 6.073 & 5.448 \\
\hline $\begin{array}{l}\text { Broad sense heritability } \\
\left(h^{2} . b\right) \%\end{array}$ & 83.02 & 91.87 & 87.09 & 83.10 & 92.48 & 95.98 & 95.25 & 95.33 & 95.55 & 65.71 & 83.56 & 97.81 & 98.93 \\
\hline $\begin{array}{l}\text { Narrow sense heritability } \\
\left(h^{2} \cdot n\right) \%\end{array}$ & 50.43 & 78.42 & 75.01 & -15.02 & 38.80 & 69.17 & 69.49 & 61.18 & 65.31 & 8.864 & 29.95 & 87.93 & 0.294 \\
\hline $\begin{array}{l}\text { Relative importance of } \\
\text { GCA \% }\end{array}$ & 60.75 & 85.35 & 86.12 & -18.07 & 41.96 & 72.07 & 72.95 & 64.60 & 68.36 & 13.49 & 35.84 & 89.90 & 0.30 \\
\hline $\begin{array}{l}\text { Relative importance of } \\
\text { SCA \% }\end{array}$ & 39.25 & 14.65 & 13.88 & 118.13 & 57.85 & 27.93 & 27.04 & 35.40 & 31.64 & 86.51 & 64.18 & 10.08 & 99.70 \\
\hline
\end{tabular}

* Relative importance of GCA $\%=\sigma^{2} \mathrm{~A} / \sigma^{2} \mathrm{G}$.

$* *$ Relative importance of SCA $\%=\sigma^{2} D / \sigma^{2} G$. 
Table 6. Mean performance of all genotypes for the agronomic and yield components character at the two years and their combined data.

\begin{tabular}{|c|c|c|c|c|c|c|c|c|c|c|c|c|c|c|c|}
\hline \multirow{2}{*}{ Genotype } & \multicolumn{3}{|c|}{ Days to heading (days) } & \multicolumn{3}{|c|}{ Plant height $(\mathrm{cm})$} & \multicolumn{3}{|c|}{ Panicles plant $^{-1}$} & \multicolumn{3}{|c|}{ Panicle length $(\mathrm{cm})$} & \multicolumn{3}{|c|}{ Panicle weight (g) } \\
\hline & $Y_{1}$ & $\mathrm{Y}_{2}$ & Comb. & $Y_{1}$ & $Y_{2}$ & Comb. & $Y_{1}$ & $\mathrm{Y}_{2}$ & Comb. & $Y_{1}$ & $Y_{2}$ & Comb. & $Y_{1}$ & $Y_{2}$ & Comb. \\
\hline \multicolumn{16}{|l|}{ CMS Lines (A): } \\
\hline IR 58025 B & 110.5 & 111.0 & 110.8 & 112.1 & 112.9 & 112.5 & 16.1 & 15.6 & 15.8 & 24.0 & 24.0 & 24.0 & 3.88 & 4.08 & 3.98 \\
\hline IR 68885 B & 95.0 & 93.8 & 94.4 & 96.6 & 97.6 & 97.1 & 20.5 & 20.4 & 20.4 & 23.4 & 23.3 & 23.3 & 3.13 & 3.25 & 3.19 \\
\hline G 46 B & 85.5 & 83.8 & 83.6 & 105.7 & 104.0 & 104.9 & 16.5 & 18.3 & 17.4 & 22.9 & 22.9 & 22.9 & 4.10 & 3.92 & 4.01 \\
\hline Large Stigma B & 78.9 & 79.0 & 79.0 & 94.1 & 93.3 & 93.7 & 14.6 & 13.7 & 14.1 & 20.5 & 19.8 & 20.1 & 3.05 & 3.06 & 3.05 \\
\hline \multicolumn{16}{|l|}{ Restorer Lines (R): } \\
\hline Giza $178 \mathrm{R}$ & 100.5 & 101.0 & 100.8 & 103.5 & 105.0 & 104.2 & 20.5 & 20.8 & 20.6 & 22.8 & 23.8 & 23.3 & 3.99 & 4.10 & 4.04 \\
\hline Giza $181 \mathrm{R}$ & 112.8 & 114.3 & 113.5 & 108.0 & 109.3 & 108.7 & 21.7 & 20.0 & 20.8 & 24.3 & 24.4 & 24.3 & 4.11 & 4.19 & 4.15 \\
\hline Giza $182 \mathrm{R}$ & 93.8 & 94.0 & 93.3 & 98.7 & 99.2 & 98.9 & 20.2 & 20.5 & 20.3 & 24.9 & 24.9 & 24.9 & 3.86 & 4.06 & 3.96 \\
\hline \multicolumn{16}{|l|}{ Hybrid combinations: } \\
\hline IR 58025 A $\quad$ /Giza 178 R & 101.5 & 102.0 & 101.8 & 103.7 & 104.3 & 104.0 & 18.8 & 20.0 & 19.4 & 25.4 & 25.7 & 25.6 & 4.89 & 4.99 & 4.94 \\
\hline /Giza $181 \mathrm{R}$ & 113.0 & 111.5 & 112.3 & 111.7 & 111.1 & 111.4 & 19.5 & 20.0 & 19.7 & 28.9 & 28.6 & 28.7 & 5.60 & 5.70 & 5.65 \\
\hline /Giza $182 \mathrm{R}$ & 100.8 & 102.5 & 101.6 & 112.0 & 104.7 & 108.3 & 19.3 & 20.4 & 19.8 & 26.8 & 26.8 & 26.8 & 4.90 & 4.69 & 4.79 \\
\hline /Giza $178 \mathrm{R}$ & 94.3 & 94.5 & 94.4 & 98.5 & 97.0 & 97.7 & 21.9 & 26.1 & 24.0 & 25.6 & 25.6 & 25.6 & 3.74 & 4.16 & 3.95 \\
\hline /Giza $181 \mathrm{R}$ & 105.3 & 105.5 & 105.4 & 99.6 & 100.1 & 100.0 & 23.3 & 23.4 & 23.4 & 26.6 & 26.5 & 26.5 & 3.57 & 3.77 & 3.67 \\
\hline /Giza $182 \mathrm{R}$ & 99.0 & 99.3 & 99.1 & 97.3 & 100.0 & 98.6 & 23.6 & 25.9 & 24.8 & 26.2 & 25.8 & 26.0 & 4.26 & 4.25 & 4.25 \\
\hline /Giza 178 R & 101.0 & 101.5 & 101.3 & 114.7 & 111.4 & 113.0 & 18.8 & 19.0 & 18.9 & 26.9 & 27.0 & 26.9 & 5.87 & 5.42 & 5.64 \\
\hline /Giza $181 \mathrm{R}$ & 103.0 & 102.5 & 102.8 & 110.1 & 110.2 & 110.2 & 20.0 & 26.1 & 23.1 & 25.3 & 25.6 & 25.5 & 3.68 & 3.79 & 3.74 \\
\hline /Giza 182 R & 99.3 & 100.3 & 99.8 & 116.9 & 115.2 & 116.0 & 19.1 & 26.6 & 22.9 & 26.0 & 26.0 & 26.0 & 5.53 & 5.34 & 5.44 \\
\hline Large Stigma /Giza 178 R & 101.0 & 101.8 & 101.4 & 108.1 & 108.0 & 108.0 & 16.9 & 18.4 & 17.6 & 25.2 & 25.5 & 25.3 & 4.77 & 4.80 & 4.79 \\
\hline /Giza 181 R & 102.5 & 102.8 & 102.6 & 109.5 & 111.8 & 110.6 & 18.5 & 18.9 & 18.7 & 26.5 & 26.5 & 26.5 & 4.06 & 4.00 & 4.03 \\
\hline /Giza $182 \mathrm{R}$ & 98.5 & 97.3 & 97.9 & 106.6 & 109.5 & 108.1 & 17.6 & 18.0 & 17.8 & 27.0 & 27.2 & 27.1 & 4.93 & 5.12 & 5.03 \\
\hline L.S.D. & 0.95 & 0.86 & 0.67 & 1.79 & 1.90 & 1.48 & 1.05 & 1.05 & 1.12 & 0.41 & 0.53 & 0.34 & 0.31 & 0.18 & 0.19 \\
\hline $1 \%$ & 1.26 & 1.15 & 0.88 & 2.38 & 2.52 & 1.96 & 1.39 & 1.40 & 1.48 & 0.55 & 0.70 & 0.45 & 0.41 & 0.24 & 0.25 \\
\hline
\end{tabular}


Cont'd. Table 6.

\begin{tabular}{|c|c|c|c|c|c|c|c|c|c|c|c|c|}
\hline \multirow{3}{*}{ Genotype } & \multicolumn{6}{|c|}{ Spikelets panicle $^{-1}$} & \multicolumn{6}{|c|}{ Field grains panicle $^{-1}$} \\
\hline & \multicolumn{3}{|c|}{ Begged } & \multicolumn{3}{|c|}{ Unbagged } & \multicolumn{3}{|c|}{ Begged } & \multicolumn{3}{|c|}{ Unbagged } \\
\hline & $Y_{1}$ & $Y_{2}$ & Comb. & $Y_{1}$ & $Y_{2}$ & Comb. & $Y_{1}$ & $Y_{2}$ & Comb. & $Y_{1}$ & $Y_{2}$ & Comb. \\
\hline \multicolumn{13}{|l|}{ CMS Lines $(A)$ : } \\
\hline IR 58025 B & 237.0 & 228.3 & 232.6 & 228.5 & 231.8 & 230.1 & 183.9 & 179.0 & 181.5 & 191.4 & 192.5 & 191.9 \\
\hline IR $68885 \mathrm{~B}$ & 144.3 & 138.5 & 141.4 & 145.9 & 145.7 & 145.8 & 116.5 & 108.0 & 112.3 & 124.3 & 121.5 & 122.9 \\
\hline G 46 B & 147.7 & 151.9 & 149.8 & 151.3 & 156.7 & 154.0 & 127.9 & 131.0 & 129.5 & 138.6 & 144.1 & 141.3 \\
\hline Large Stigma B & 115.2 & 116.4 & 115.8 & 117.4 & 119.4 & 118.4 & 100.0 & 101.7 & 100.8 & 107.6 & 110.7 & 109.2 \\
\hline \multicolumn{13}{|l|}{ Restorer Lines (R): } \\
\hline Giza $178 \mathrm{R}$ & 172.1 & 162.0 & 167.1 & 172.4 & 173.1 & 172.8 & 140.3 & 129.4 & 134.9 & 149.6 & 147.3 & 148.4 \\
\hline Giza $181 \mathrm{R}$ & 164.6 & 162.4 & 163.5 & 165.4 & 163.5 & 164.5 & 135.1 & 131.5 & 133.3 & 147.4 & 148.4 & 147.9 \\
\hline Giza $182 \mathrm{R}$ & 153.9 & 157.5 & 155.7 & 153.5 & 158.1 & 155.8 & 132.0 & 134.5 & 133.3 & 142.8 & 145.9 & 144.4 \\
\hline \multicolumn{13}{|l|}{ Hybrid combinations: } \\
\hline IR 58025 A /Giza 178 R & 226.1 & 220.7 & 223.4 & 226.6 & 225.9 & 226.2 & 181.7 & 173.5 & 177.6 & 195.5 & 183.2 & 189.4 \\
\hline /Giza $181 \mathrm{R}$ & 215.8 & 208.2 & 212.0 & 213.2 & 214.6 & 213.9 & 174.6 & 168.3 & 171.4 & 191.3 & 175.6 & 183.5 \\
\hline /Giza $182 \mathrm{R}$ & 171.2 & 177.2 & 174.2 & 181.1 & 180.4 & 180.7 & 137.3 & 143.4 & 140.4 & 159.9 & 155.0 & 157.4 \\
\hline IR 68885 A /Giza 178 R & 131.9 & 134.8 & 133.4 & 131.9 & 137.9 & 134.9 & 105.1 & 104.3 & 104.7 & 114.7 & 112.6 & 113.7 \\
\hline /Giza $181 \mathrm{R}$ & 135.3 & 139.2 & 137.2 & 135.5 & 133.5 & 134.5 & 104.8 & 108.4 & 106.6 & 109.9 & 107.7 & 108.8 \\
\hline /Giza $182 \mathrm{R}$ & 141.7 & 146.9 & 144.3 & 147.3 & 148.7 & 148.0 & 111.1 & 113.4 & 112.2 & 123.7 & 120.4 & 122.1 \\
\hline G 46 A /Giza 178 R & 206.4 & 198.0 & 202.2 & 198.6 & 205.0 & 201.8 & 183.9 & 171.0 & 177.5 & 189.4 & 190.4 & 189.9 \\
\hline /Giza $181 \mathrm{R}$ & 170.4 & 170.5 & 170.4 & 170.2 & 168.6 & 169.4 & 137.8 & 136.9 & 137.3 & 143.8 & 136.5 & 140.2 \\
\hline /Giza 182 R & 183.7 & 183.1 & 183.4 & 185.5 & 182.6 & 184.0 & 151.9 & 145.3 & 148.6 & 163.9 & 154.2 & 159.1 \\
\hline Large Stigma/Giza $178 \mathrm{R}$ & 202.7 & 200.2 & 201.5 & 194.7 & 201.2 & 198.0 & 161.0 & 160.2 & 160.6 & 165.5 & 168.5 & 167.0 \\
\hline /Giza $181 \mathrm{R}$ & 185.6 & 190.1 & 187.8 & 174.5 & 187.7 & 181.1 & 141.2 & 146.2 & 143.7 & 136.2 & 147.6 & 141.9 \\
\hline /Giza $182 \mathrm{R}$ & 212.8 & 215.2 & 214.0 & 210.5 & 215.7 & 213.1 & 177.5 & 183.7 & 180.6 & 178.8 & 189.3 & 184.0 \\
\hline L.S.D. & 6.76 & 6.55 & 4.75 & 7.17 & 7.43 & 4.97 & 7.08 & 5.97 & 4.79 & 7.14 & 6.41 & 5.12 \\
\hline $1 \%$ & 8.99 & 8.71 & 6.27 & 9.54 & 9.88 & 6.56 & 9.42 & 7.94 & 6.33 & 9.50 & 8.53 & 6.77 \\
\hline
\end{tabular}


Cont'd. Table 6.

\begin{tabular}{|c|c|c|c|c|c|c|c|c|c|c|c|c|}
\hline \multirow{3}{*}{ Genotypes } & \multicolumn{6}{|c|}{ Spikelet fertility \% } & \multirow{2}{*}{\multicolumn{3}{|c|}{1000 -grain weight $(\mathrm{g})$}} & \multirow{2}{*}{\multicolumn{3}{|c|}{ Yield t/ha }} \\
\hline & \multicolumn{3}{|c|}{ Begged } & \multicolumn{3}{|c|}{ Unbagged } & & & & & & \\
\hline & $Y_{1}$ & $\mathrm{Y}_{2}$ & Comb. & $Y_{1}$ & $Y_{2}$ & Comb. & $Y_{1}$ & $Y_{2}$ & Comb. & $Y_{1}$ & $Y_{2}$ & Comb. \\
\hline \multicolumn{13}{|l|}{ CMS Lines $(A):$} \\
\hline IR 58025 B & 77.7 & 78.5 & 78.1 & 83.7 & 83.1 & 83.4 & 22.95 & 22.40 & 22.68 & 8.75 & 8.89 & 8.82 \\
\hline IR 68885 B & 80.7 & 78.1 & 79.4 & 85.2 & 83.5 & 84.3 & 22.43 & 22.40 & 22.41 & 8.61 & 8.53 & 8.57 \\
\hline G 46 B & 86.7 & 86.2 & 86.5 & 91.7 & 92.0 & 91.8 & 28.10 & 27.82 & 27.96 & 8.19 & 8.19 & 8.19 \\
\hline Large Stigma B & 86.7 & 87.4 & 87.1 & 91.7 & 92.7 & 92.0 & 30.50 & 31.02 & 30.76 & 5.48 & 6.09 & 5.78 \\
\hline \multicolumn{13}{|l|}{ Restorer Lines $(\mathrm{R})$ : } \\
\hline Giza 178 R & 81.6 & 80.0 & 80.8 & 86.8 & 85.1 & 86.0 & 22.30 & 22.12 & 22.21 & 10.63 & 10.40 & 10.52 \\
\hline Giza $181 \mathrm{R}$ & 82.2 & 81.0 & 81.6 & 89.1 & 90.8 & 90.0 & 27.65 & 27.33 & 27.49 & 10.54 & 10.55 & 10.55 \\
\hline Giza $182 \mathrm{R}$ & 85.8 & 85.4 & 85.6 & 93.1 & 92.3 & 92.7 & 27.15 & 27.11 & 27.13 & 10.46 & 10.38 & 10.47 \\
\hline \multicolumn{13}{|l|}{ Hybrid combinations: } \\
\hline IR 58025 A /Giza 178 R & 80.4 & 79.4 & 79.9 & 86.3 & 81.1 & 83.7 & 25.41 & 25.41 & 25.41 & 11.81 & 12.17 & 11.99 \\
\hline /Giza $181 \mathrm{R}$ & 80.9 & 80.9 & 80.9 & 89.7 & 81.8 & 85.8 & 28.77 & 28.64 & 28.71 & 12.78 & 12.91 & 12.84 \\
\hline /Giza $182 \mathrm{R}$ & 80.2 & 81.1 & 80.6 & 88.2 & 86.0 & 87.1 & 26.87 & 27.20 & 27.03 & 10.89 & 10.80 & 10.85 \\
\hline IR 68885 A /Giza 178 R & 79.7 & 77.4 & 78.5 & 87.0 & 81.7 & 84.3 & 24.21 & 23.99 & 24.10 & 11.76 & 11.80 & 11.78 \\
\hline /Giza $181 \mathrm{R}$ & 77.5 & 77.9 & 77.7 & 81.1 & 80.7 & 80.9 & 26.28 & 26.44 & 26.36 & 12.01 & 10.61 & 11.31 \\
\hline /Giza 182 R & 77.6 & 77.2 & 77.4 & 84.0 & 81.4 & 82.7 & 26.20 & 26.26 & 26.33 & 8.69 & 8.29 & 8.48 \\
\hline /Giza 178 R & 89.1 & 86.4 & 87.7 & 95.4 & 92.9 & 94.2 & 28.30 & 27.85 & 28.07 & 13.01 & 12.96 & 12.99 \\
\hline /Giza $181 \mathrm{R}$ & 80.9 & 80.3 & 80.6 & 84.5 & 81.0 & 82.8 & 28.76 & 28.86 & 28.83 & 11.41 & 11.34 & 11.38 \\
\hline /Giza $182 \mathrm{R}$ & 82.7 & 79.3 & 81.0 & 88.4 & 84.5 & 86.4 & 30.54 & 30.58 & 30.56 & 12.38 & 12.90 & 12.64 \\
\hline Large Stigma/Giza 178 R & 79.4 & 79.2 & 79.3 & 85.0 & 83.7 & 84.4 & 29.55 & 29.60 & 29.57 & 10.23 & 9.89 & 10.06 \\
\hline /Giza $181 \mathrm{R}$ & 76.1 & 76.9 & 76.5 & 78.1 & 78.7 & 78.4 & 30.16 & 30.25 & 30.20 & 7.46 & 7.72 & 7.59 \\
\hline /Giza $182 \mathrm{R}$ & 83.4 & 85.4 & 84.4 & 84.9 & 87.7 & 86.3 & 30.82 & 30.75 & 30.88 & 11.53 & 11.82 & 11.62 \\
\hline L.S.D. & 2.66 & 1.83 & 1.57 & 1.62 & 1.90 & 1.52 & 0.39 & 0.54 & 0.32 & 0.40 & 0.34 & 0.31 \\
\hline $1 \%$ & 3.53 & 2.44 & 2.08 & 2.16 & 2.53 & 2.01 & 0.51 & 0.71 & 0.42 & 0.53 & 0.46 & 0.41 \\
\hline
\end{tabular}


Table 7. Percentage of heterosis of the $12 \mathrm{~F}_{1}$ hybrids over standard variety (SH) for agronomic and yield and its components characters (combined analysis).

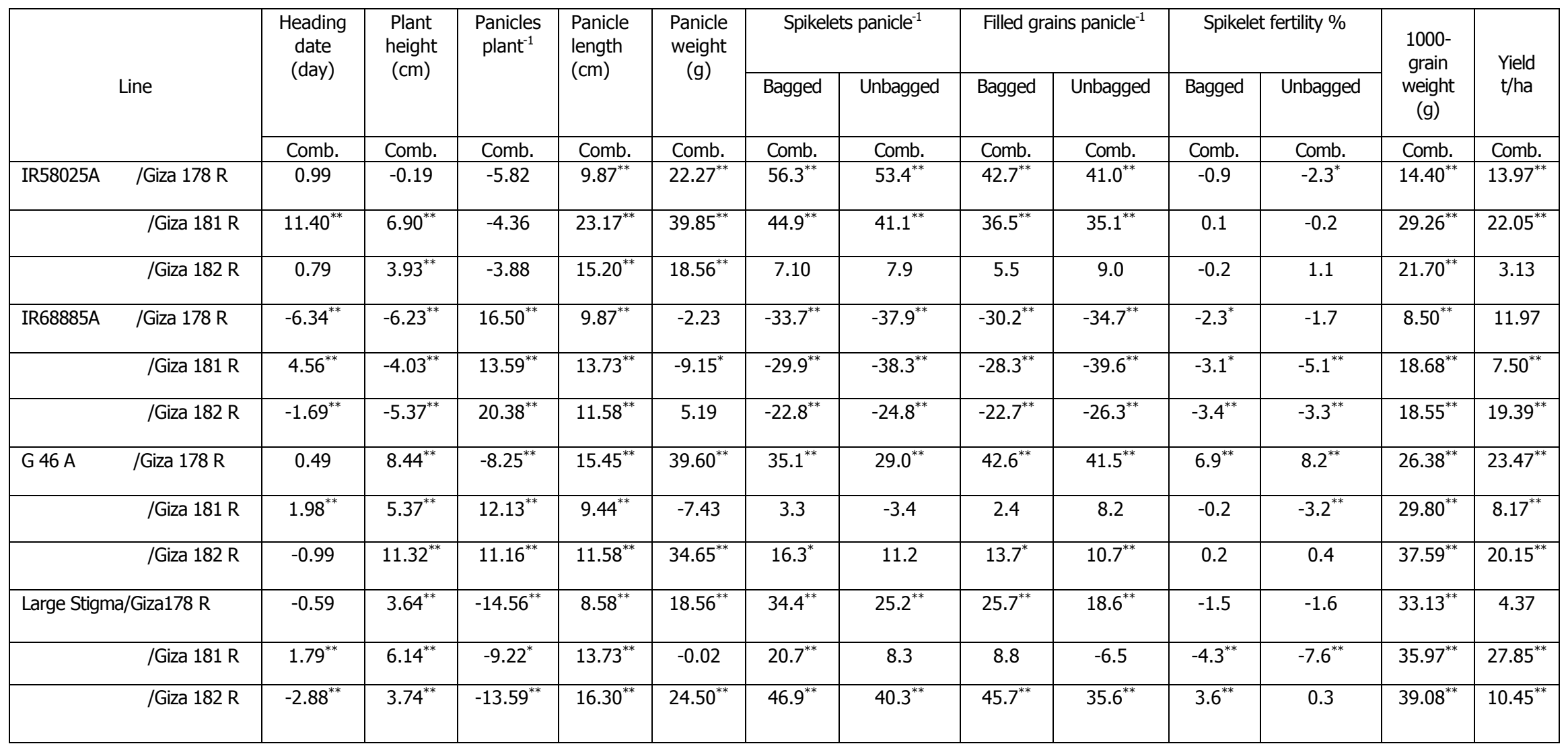

* and ** significant at $5 \%$ and $1 \%$ probability levels, respectively. 
Highest panicle weight was observed in " IR58025A / Giza 181R" (5.65g) and lowest in "IR68885A / Giza 181R" (3.67g). The trait spikelets pacinle ${ }^{-1}$ was varied from 134.5 spikelet (in IR58025A / Giza 181R) to 226.2 spikelets ( in IR58025A / Giza 178R). Filled grains panicle was exhibited highest in "G46A / Giza178R" (189.9) and lowest in "IR68885A / Giza 181R" (108.8). The hybrid rice combination G46A / Giza178R was exhibited highest spikelet fertility (94.2\%). Hybrid large stigma A / Giza $182 \mathrm{R}$ was exhibited highest 1000-grain weight (30.88g), while the hybrid rice combinations, " IR68885A / Giza 178R" and "IR58025A / Giza 178R" exhibited lowest 1000- grain weight ( 24.10 and $25.41 \mathrm{~g}$, respectivilly).

Six rice hybrids were performed better yield than high yielding check variety Giza 178. Higher yield of rice hybrids resulted from their increased spikelet number and to some extent increased grain weight, which enhanced the sink capacity. Peng et al. (2003) reported that the average yield of $F_{1}$ hybrid rice was $17 \%$ higher than that of indica inbreds. Yield ton ha ${ }^{-1}$ was recorded highest in " G46A / Giza 178R (12.99 ton $\left.\mathrm{ha}^{-1}\right)$ and lowest in " large stigma A/ Giza 181R " (7.59 ton ha $\left.{ }^{-1}\right)$. Highest yield was produced by "G46A / Giza 178R"(12.99 ton ha-1) followed by "IR58025A / Giza 181R" (12.84 ton ha ${ }^{-1}$ ) and " G46A / Giza 182R" ( 12.64 ton ha ${ }^{-1}$ ).

\section{Standard Heterosis}

Heterosis is the measure of the deviation of the $F_{1}$ hybrids over its parents and the check variety. It was estimated versus over check variety or standard heterosis(SH). In commercial exploitation positive standard heterosis alone holds relevance as a hybrid may be superior to commercially cultivated best variety (Giza 178). The standard heterosis is especially important because the hybrid to be released is expected to out perform the existing superior local variety or hybrid. The data for standard heterosis in 12 hybrid combination are presented in Table (7). Evaluation based on the standard heterosis revealed that three hybrids recorded significant negative standard heterosis for early heading data. The hybrids IR68885A / Giza 178R, large stigma A/ Giza 182R and IR68885 A / Giza 182R and IR68885A / Giza $182 R$, were the earliest to flower within $94.4,97.9$ and 99.1 days respectively. These findings indicated that heterosis effects can be used to get earliness in rice hybrids (El-Mowafi 2001b, El-Mowafi and Abo-Shousha 2003 and El-Mowafi et al. 2005). Two hybrids recorded negative standard heterosis for short stature plant, five rice hybrid recorded positive $\mathrm{SH} \%$ for panicles plant ${ }^{-1}$, all the 12 for panicle length, seven for panicle weight, five hybrids recorded positive highly significant standard heterosis for spikelets panicle ${ }^{-1}$, six for filled grains panicle ${ }^{-1}$, one hybrid combination for spikelet fertility $\%$ bagged and unbagged panicles and all 12 for 1000-grain weight. However, positive and significant standard heterosis was recorded in 10 hybrid combinations for 
grain yield (ton ha-1). The outstanding hybrid combinations for grain yield were large stigma A / Giza 181R (27.85\%) with significant standard heterosis for panicle length, spikelets panicle ${ }^{-1}$ and 1000-grain weight. In the mean time, G46A / Giza 178R $(23.47 \%)$ gave significant desirable heterosis for panicle length $(15.45 \%)$, panicle weight ( $39.60 \%)$, spikelets panicle $(35.10 \%)$, filled grains panicle $(42.60 \%)$, spikelet fertility (6.9\%) and 1000-grain weight (26.38\%). Also, IR58025A / Giza 181R with significant standard heterosis for panicle length $(23.17 \%)$, panicle weight $(39.85 \%)$, spikelets panicle $(44.90 \%)$, filled grains panicle ${ }^{-1}$ (36.5\%) and 1000-grain weight (29.36\%). G46A / Giza 182R (20.15\%) gave significant desirable standard heterosis for panicles plant $^{-1}(11.16 \%)$, panicle length $(11.58 \%)$, panicle weight $(34.65 \%)$, spikelets panicle $^{-1}(16.30 \%)$, filled grains panicle ${ }^{-1}(13.70 \%)$ and 1000 -grain weight (37.59\%).

\section{REFERENCES}

1. AbdAllah, R. M. 2008. Genetical and morphological studies on environmental genetic and cytoplasmic male sterility lines in rice. M.Sc. Thesis, Fac. of Agric. Mansoura Univ. Egypt.

2. Babu, S.S. and P.S. Reddy 2002. Combining ability analysis in rice (Oryza sativa L.). Research-on-Crops. 3(3): 592-598.

3. Batlett, N. S. 1937. Properties of sufficiency and statistical tests. Proc. Roy Soc. Londen, Series A, $160:$ 268-282.

4. Bastawisi, A.O., H.F. El-Mowafi, M.I. Abo Youssef, A.E. Draz, I.R. Aidy, M.A. Maximos and A.T. Badawi 2003. Hybrid rice research and development in Egypt. $4^{\text {th }}$ Int. Symposium on Hybrid Rice 14-17 May 2002, Hanoi, Vietnam.

5. El-Badawi 2009. Genetic Studies on Improvement of Some Restorer Lines for Cytoplasmic Genetic Male Sterile (CMS) Lines in Rice. M.Sc. Thesis, Fac. of Agric. Mansoura Univ. Egypt.

6. El-Diasty, Z. M., H. F. El-Mowafi, M. S. Hamada and R. M. Abdallah 2008. Genetic studies on photo-thermo-sensitive genic male sterility (P/TGMS) and its utilization in rice breeding. J. Agric. Sci. Mansoura Univ., 33(5): 3391-3404.

7. El-Mowafi, H.F. 2001a. Study on heterosis in hybrid rice under Egyptian conditions. Egypt. J. Appl. Sci., 16(2), 52-63.

8. El-Mowafi, H.F. 2001b. Combining ability analysis for some cytoplasmic male sterile and restorer rice lines under Egyptian conditions. Egypt. J. Appl. Sci., 16(2): 25-51. 
9. El-Mowafi, H.F. and A.A. Abou-Shousha 2003. Combining ability and heterosis analysis of diverse CMS lines in hybrid rice. J. Agric. Res. Tanta Univ., 29(1): 106127.

10. El-Mowafi, H.F., A.O. Bastawisi, M.I. Abou Youssef and F.U. Zaman 2005. Exploitation of rice heterosis under Egyptian conditions. Egypt. J. Agric. Res. 389 (5A): 143-166.

11. El-Mowafi, H.F. 2006. Genetic and combining ability analysis of grain quality characters in hybrid rice, J. Agric. Sci. Mansoura Univ.,31 (8): 4929-4945.

12. El-Refaee, Y.Z.E. 2002. Genetical and biochemical studies on heterosis and combining ability in rice. M.Sc. Thesis, Fac. Agric., Tanta Univ., Kafr El-Sheikh, Egypt.

13. Kempthorne, O. 1957. An Introduction to Genetic Statistics. John Wiley and Sons Inc., New York, 458-471.

14. Neelam, S., M.S. Ramesha , T.D. Reddy and A.Sankar 2009. Study of heterosis by utilizing male sterility restoration system in rice (Oryza sativa L.). J.Rice Res., 2: $110-120$

15. Panse, V.G. and P.V. Sukhatme 1954. Statistical Methods for Agricultural Workers. Indian Council of Agric. Res., New Delhi, 227 p.

16. Peng, S., J. Yang, R.C. Laza, A.L. Sanico, R.M. Visperas and T.T. Song. 2003. Physiological bases of heterosis and crop management strategies for hybrid rice in the tropics. In: Virmani, S.S., C.X. Mao and B. Dardy (eds.) Hybrid Rice for Food Security, Poverty Alleviation, and Environment Protection. Proc. 4th Intl. Symp. Hybrid Rice, 14-17 May 2002, Hanoi, Vietnam. Los Baños (Philippines): Intl. Rice Res. Inst. pp. 153-170.

17. Rastogi A., B.K. Mishra, M. Srivastava, A. Siddiqui, S. Shukla 2011. Biplot approach foridentification of heterotic crosses in linseed (Linum usitatissimum L.) J. of Botany 11, 1-7.

18. Yan, S., S. Wen, Z. Li, J. Wan, Y. Tian and C. Liu. 2000. Study on the combining ability of indica two-line hybrid rice. J. Huozhong Agric. Univ., 19: 204-208. 


\section{دراسات القدرة علي الإيتّلاف وقوة الهجين بإستخدام مصادر عقم ذكري سيتويلازمي مختلفة في تريبة الأرز الهجين الأنين}

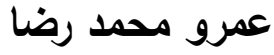

مركز البحوث والتنديب في الارز - معهُ بحوث الدحاصيل الحقلية - مركز البحوث الزراعية.

أجريت هذه الدراسة خلال موسمي زراعة الأرز با •r و ع ا. ب بالمزرعة البحثية لمحطة البحوث الزراعية بسخا - كفر الثيخ - مصر ، وذللك لدراسة أهمية التباين الوراثي و أهمية كل من القدرة العامة والخاصة علي الإئتلاف و التفاعل بين موسمي النمو كظروف بيئية مختلفة ، وكذلك تقدير القياسات الوراثية ودرجة التوريث بالمعني الواسع والضيق. وكذلك قوة الهجين للصفات الحقلية وصفات المحصول ومكوناته في تربية الأرز الهجين بإستخدام سلالات العقم الذكري السيتوبلازمي الوراتي الثلاتي من مصادر عقم مختلفة ومنتوعة وكذلك ثلاثة آباء مصرية معيدة للخصوبة وهي

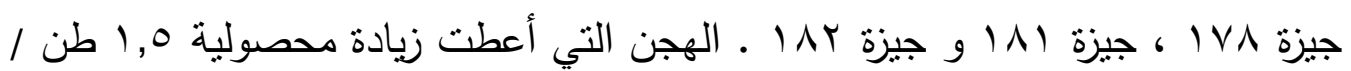

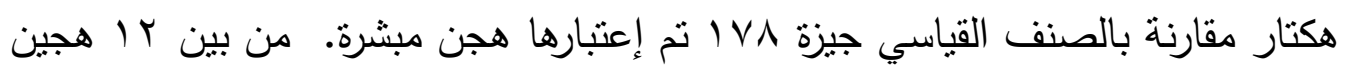
تم اختبارهم ، وجد أن ستة منها أعطت أفضل النتائج بالنسبة لصفة المحصول ويتراوح

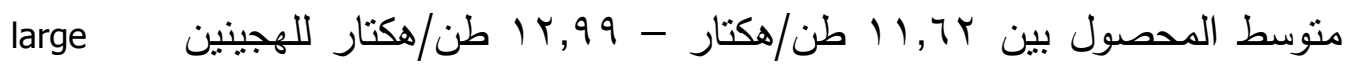
متوكانت متوسط الزيادة المحصولية مقارنة

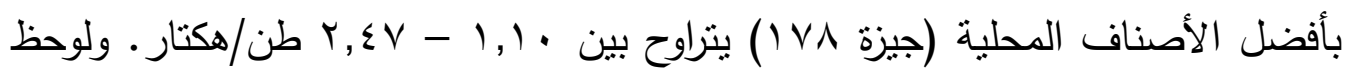

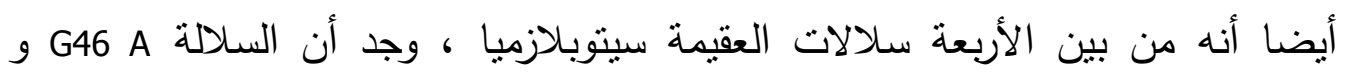
كانتا أفضل السلالات من حيث القدرة العامة علي الإئتلاف وذللك لصفة IR58025 A محصول الحبوب وكان الصنف المعيد للخصوبة Giza 178R هو الأفضل بين الكثافات المستخدمة في الدراسة لصفة محصول الحبوب وكذلك معظم الصفات تحت الدراسة. 